\title{
On using compressibility to detect when slime mould completed computation
}

\author{
Andrew Adamatzky and Jeff Jones \\ Unconventional Computing Centre, \\ University of the West of England, UK
}

November 26, 2014

\begin{abstract}
Slime mould Physarum polycephalum is a single cell visible by an unaided eye. The slime mould optimises its network of protoplasmic tubes in gradients of attractants and repellents. This behaviour is interpreted as computation. Several prototypes of the slime mould computers were designed to solve problems of computation geometry, graphs, transport networks and to implement universal computing circuits. Being a living substrate the slime mould does not halt its behaviour when a task is solved but often continues foraging the space thus masking the solution found. We propose to use temporal changes in compressibility of the slime mould patterns as indicators of the halting of the computation. Compressibility of a pattern characterises the pattern's morphological diversity, i.e. a number of different local configurations. At the beginning of computation the slime explores the space thus generating less compressible patterns. After gradients of attractants and repellents are detected the slime spans data sites with its protoplasmic network and retracts scouting branches, thus generating more compressible patterns. We analyse the feasibility of the approach on results of laboratory experiments and computer modelling. Keywords: Compressibility, slime mould, computation
\end{abstract}

\section{Introduction}

Slime mould Physarum polycephalum belongs to the species of order Physarales, subclass Myxogastromycetidae, class Myxomycetes, division Myxostelida. The slime mould is commonly found in its vegetative form, a single cell with a myriad of diploid nuclei - the plasmodium. The plasmodium behaves and moves as a giant amoeba. It feeds on bacteria, spores and other microbial creatures and micro-particles [27]. During its foraging behaviour the plasmodium spans scattered sources of nutrients with a network of protoplasmic tubes. The plasmodium optimises its protoplasmic network that covers all sources of nutrients and guarantees robust and quick distribution of nutrients in the plasmodium's body. Plasmodium's foraging behaviour can be interpreted as a computation: data are represented by the spatial pattern of attractants and repellents, and results are represented by the structure of the protoplasmic network [20, 3]. Plasmodium can solve computational problems with natural parallelism, e.g. 
related to shortest path and hierarchies of planar proximity graphs, execution of logical computing schemes and natural implementation of spatial logic and process algebra [3].

We developed a concept and designed a series of experimental laboratory prototypes of computing devices - Physarum machines [3] — based on P. polycephalum. A Physarum machine is a programmable amorphous biological computing device experimentally implemented in plasmodium of $P$. polycephalum. A Physarum machine is programmed by configurations of repelling and attracting gradients. See detailed analysis of Physarum machines in [3]. A mechanics of Physarum machines is based on the following unique features of $P$. polycephalum. Physarum is a reaction-diffusion excitable medium encapsulates in an elastic growing membrane (excitation is triggered by chemical, physical and mechanical stimuli). Physarum may be regarded as a living micro-manipulation and micro-fluidic transport device. Physarum represents results of computation by configuration of its body: topology of the Physarum network is mediated by its environment, the distribution and gradients of chemo-attractants and repellents. Physarum is sensitive to illumination and therefore allows for parallel input of information.

Physarum machines are proved to be most successful biological substrates in solving problems of computation geometry, optimisation and logic. Their only 'faults' are that the machines are slow and never stop. Speed of the Physarum machines can not be improved cause it is limited by speeds of bio-chemical and physiological processes. However, the halting of computation by the slime mould machines can be dealt with. Physarum machines, as most unconventional do, represent results of the computation by changing their morphology. Changes of the morphology can be detected by using one of the complexity measures. Potential compexity measures to employ could be Shannon entropy, Simpson index, morphological diversity, generative complexity or Derrida coefficients $[12,26,4,8]$. These measures are proved to be useful when characterising behaviour of cellular automata (spatially expended non-linear discrete systems) and selecting cell-state transition rules responsible for generation of travelling quanta of information (gliders) [7, 4]. However, calculating these measures on images of $P$. polycephalum could be a time consuming process, which might not match requirements of experimental laboratory setups.

There is a complexity measures which can be calculated quickly on twodimensional images yet grasps all feature of space-time dynamics detected by the measures discussed above. This is compressibility. Typically Lempel-Ziv complexity is used to measure of compressibility, this is a classical and now widespread data compression algorithm $[28,12,23]$. In previous studies in cellular automaton complexity we found that the compressibility performs similarly well as Shannon entropy, Simpson index and morphological diversity in detecting when spatially extended non-linear system undergoes substantial changes in its evolution $[26,7,4,8]$. For example, in cellular automata we can detect formation of travelling localisations, propagating patterns, stable states and cycles $[7,23]$. The compressibility was also well used in analysis of living systems, e.g. EEG signals [11, 1] and DNA sequences [25], and classification of spike trains [10]. Thus we can propose that the compressibility will work well in predictive analysis of the slime mould behaviour. 


\section{Method}

\section{$2.1 \quad$ Experimental}

Experiments analysed in presented were previous described in details in $[2,2,5]$, thus we only mention key facts. Plasmodium of $P$. polycephalum was cultivated in large $(20 \times 30 \mathrm{~cm})$ plastic boxes and fed with oat flakes. Experiments on approximation of spanning trees, transport network and maze solving were undertaken on non-nutrient substrate: 2\% agar (Select Agar, Sigma Aldrich). Experiments on approximation of a Voronoi diagram were done on nutrient substrate: 2\% cornmeal agar (Fluka Analytical). In experiments with Voronoi diagram all data points were represented by oat flakes colonised the slime mould. In all other experiments discussed here all but one data points were virgin oat flakes, and the single data point was a site of inoculation. In experiments with maze solving we used plastic mazes (Tesco's Toy Mazes, Tesco Plc), $70 \mathrm{~mm}$ diameter with $4 \mathrm{~mm}$ wide and $3 \mathrm{~mm}$ deep channels. Experimental containers were scanned with Epson Perfection 4490.

\section{$2.2 \quad$ Modelling}

For the computational modelling of slime mould compressibility we employ the multi-agent model introduced in [15]. An overview of the approach can be found in [16]. In the multi-agent approach the Physarum plasmodium is approximated by a population of simple mobile particles. Each particle corresponds to a hypothetical unit of gel/sol structure within the Physarum plasmodium. Particles sense and deposit a generic chemo-attractant substance within a 2D diffusive lattice. The current pattern of the particle population represents both the structure and flux within the plasmodium. Particles orient themselves towards the locally strongest source of attractant, as sampled by its three offset sensors. Each particle attempts a movement forwards in its current orientation and deposits attractant at the new site if the movement is successful. The offset sensors effect an indirect and local coupling of the particles and the movement of a particle attracts nearby particles, forming an auto-catalytic process where self-organised networks spontaneously form and minimise in shape. The model is notable for its quasi-physical emergent behaviours including network adaptation and minimisation. The architecture of a single particle is given in Fig. 1. Particle parameters Sensor Angle (SA), Rotation Angle (RA) generate a complex range of Turing-type patterns [14] and the Sensor Offset distance (SO) acts as a pattern scaling parameter.

For the Voronoi diagram experiments we used the procedure described in [17]. For the spanning tree experiments we set SA and RA to $45^{\circ}$ and $\mathrm{RA}^{\circ}$ with SO 13. Attractants were projected onto the diffusive lattice at 25.5 units (arbitrary) per step. To reproduce the suppression of diffusion from nutrient sites on engulfment by the model plasmodium we reduced projection to 0.255 if there were agent particles within a $7 \times 7$ region of nutrient sites. Diffusion of nutrient stimuli was implemented by a mean filter kernel of size $7 \times 7$ and diffusion was weakly damped by multiplying the mean kernel result by 0.99 per scheduler step. Agent particles deposited 0.8 units per successful movement step. Diffusion of particle trails was implemented by a simple mean filter of kernel size $3 \times 3$ and diffusion distance of particle trails was strongly damped 


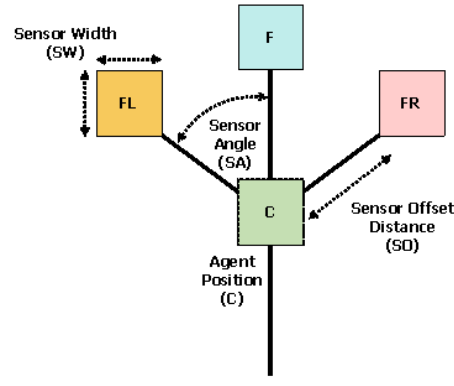

(a)

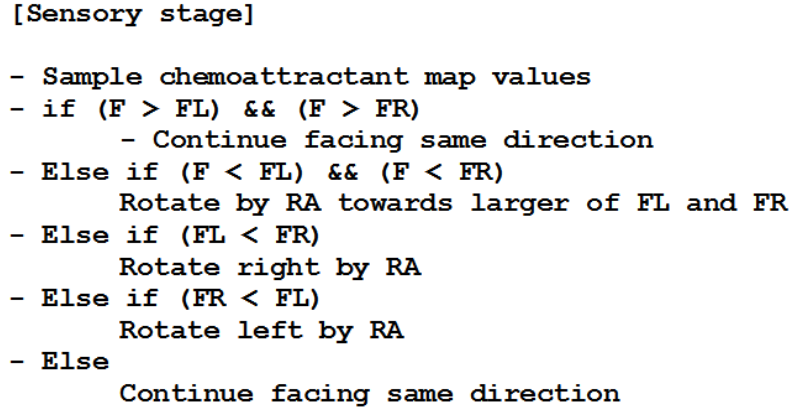

(b)

Figure 1: Single agent particle. (a) agent morphology showing position ' $\mathrm{C}$ ' and three forward biased sensors 'FL', 'F' and 'FR', (b) agent sensory algorithm governing orientation.

by multiplying by 0.9 per scheduler step.

Growth and shrinkage of the particle population is implemented as follows. If there are 1 to 20 particles in a $9 \times 9$ neighbourhood of a particle, and the particle has moved forwards successfully, the particle attempts to spawn a new particle if there is a space available at a randomly selected empty location in the immediate $3 \times 3$ neighbourhood surrounding the particle. The dependence on successful movement ensures that population growth occurs only in response to the presence of nutrient stimuli. When all nutrients have been discovered, their projection of attractants is suppressed, causing the population to automatically shrink in size using the following test. If there are 0 to 24 particles in a $5 \times 5$ neighbourhood of a particle the particle survives, otherwise it is deleted. Deletion of a particle leaves a vacant space at this location which is filled by nearby particles, causing the collective to shrink slightly. As the process continues the population shrinks and adapts its morphology to the configuration of stimuli. The frequency of testing for particle division and particle removal was every 3 scheduler steps.

\subsection{Expressing compressibility}

We express compressibility $s(t)$ of a system as a ratio of the size $f\left(m^{t}\right)$ of the compressed image $m^{t}$ of the system to the compressed size $f\left(m^{1}\right)$ of the start image $m^{1}$ taken at the beginning, $t=1$ of experiment: $s(t)=f\left(m^{t}\right) / f\left(m^{1}\right)$. Typical compression utilities, like UNIX utility GZIP work well to represent the measures of compressibility [18, 19, 9].

\section{Results}

We illustrate our approach on three problems: Voronoi diagram, spanning tree and path in a maze. The Voronoi diagram problem demonstrates who compressibility changes when almost the whole space is covered by the slime mould. The spanning problem illustrates formation of the graph and associated changes 


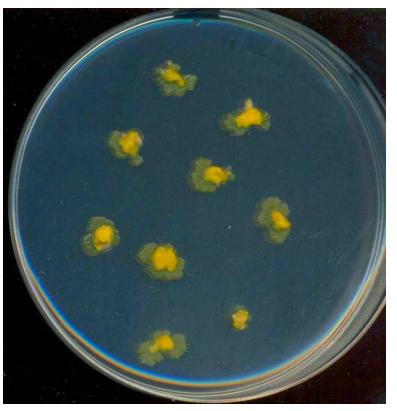

(a) $12 \mathrm{~h}$

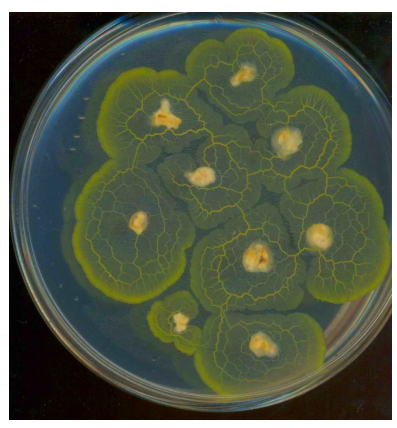

(c) $36 \mathrm{~h}$

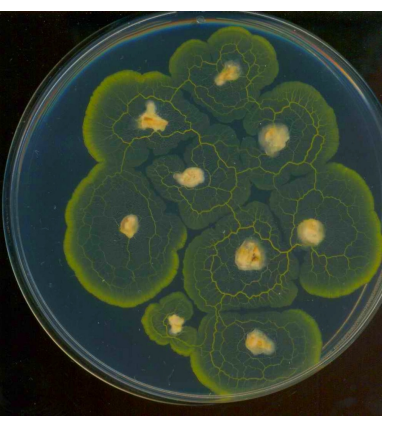

(d) $48 \mathrm{~h}$

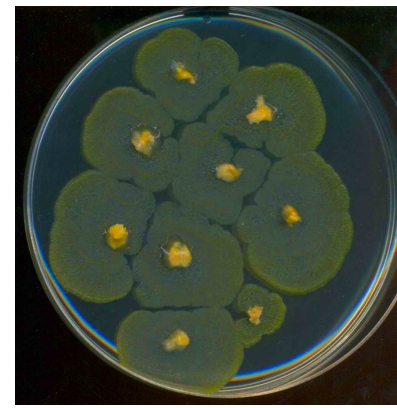

(b) $24 \mathrm{~h}$

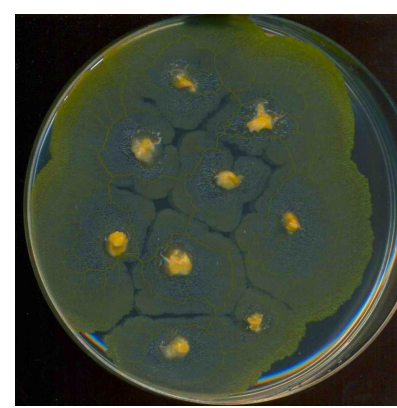

(e) $60 \mathrm{~h}$

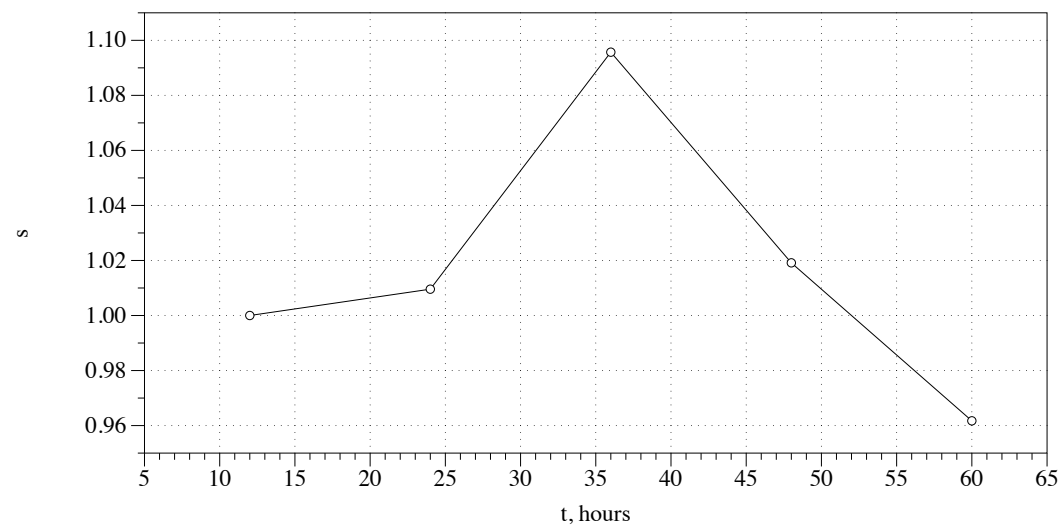

(f)

Figure 2: Physarum approximate Voronoi diagram [2]. (a-e) Photographs of the experimental setup taken in $12 \mathrm{~h}$ intervals. See details of setup in [2]. (f) Dynamic of compressibility $s(t)$.

in complexity. The path in a maze problem shows how compressibility reflects formation of scouting branches.

\subsection{Voronoi diagram}

Let $\mathbf{P}$ be a nonempty finite set of planar points. A planar Voronoi diagram of the set $\mathbf{P}$ is a partition of the plane into such regions, that for any element 
of $\mathbf{P}$, a region corresponding to a unique point $p$ contains all those points of the plane which are closer to $p$ than to any other node of $\mathbf{P}$. A unique region $\operatorname{vor}(p)=\left\{z \in \mathbf{R}^{2}: d(p, z)<d(p, m) \forall m \in \mathbf{R}^{2}, m \neq z\right\}$ assigned to point $p$ is called a Voronoi cell of the point $p$. The boundary of the Voronoi cell of point $p$ is built of segments of bisectors separating pairs of geographically closest points of the given planar set $\mathbf{P}$. A union of all boundaries of the Voronoi cells determines the planar Voronoi diagram: $\operatorname{VD}(\mathbf{P})=\cup_{p \in \mathbf{P}} \partial \operatorname{vor}(p)$ [13].

Planar data points $\mathbf{P}$ are represented by oat flakes colonised by Physarum. Physarum approximates the Voronoi diagram on a nutrient agar. Therefore it propagates as omnidirectional disc from each site of its inoculation (Fig. 2a). When two waves, originated from geographically neighbouring sites of inoculation, meet with each other they stop propagation (Fig. 2b). Thus loci of substrate unoccupied by Physarum represent edges of the Voronoi diagram. By calculation $s(t)$ of the experimental images (Fig. 2f) we found that sizes of images grow initially. They reach their maximum values by $36 \mathrm{~h}$ of experiment, when majority of the bisectors are formed (Fig. 2c). Then the sizes of images start declining (Fig. 2d). They reach their minimum size by $60 \mathrm{~h}$ when Physarum halts it propagation (Fig. 2e).

Using the model plasmodium we repeated the procedure described in [17] where oat flakes representing planar data points were inoculated with particles (Fig. 3, white regions) and nutrient-rich background substrate was represented by grey colour. The model population propagates outwards in an omnidirectional disc pattern (Fig. 3b-c), attracted by the stimulus from the background substrate. The Voronoi bisectors in the model population are represented by dense regions of particle occupancy (Fig. 3e). The graph in Fig. 3g of $s(t)$ indicates that the filesize increases as the individual inoculation sites grow. As the Voronoi bisectors begin to emerge (Fig. 3d), the curve begins to flatten out and the filesize then reduces as the bisectors form completely and ultimately merge. This suggests that the plateau in growth of the population (and filesize) may indicate the time when the computation of the Voronoi diagram is approximated.

\subsection{Spanning trees}

The Euclidean minimum spanning tree [21] is a connected acyclic graph which has minimum possible sum of edges' lengths. Physarum computes spanning tree on a non-nutrient substrate (Fig. 4). Planar points of the data set are represented by virgin oat flakes, not colonised by the slime mould. One of the data point is assigned to be a root, it is represented by oat flake colonised by the Physarum. In $12 \mathrm{~h}$ after inoculation the slime mould propagates along gradients of chemo-atractants towards virgin oat flake closest to the site of Physarum inoculation (Fig. 4a). Then the slime mould continues colonising oat flakes until all data points are spanned by protoplasmic tubes (Fig. 4b-e). Formation of densely branching active growing zones is reflected in increase of image size (Fig. 4b). The more oat flakes (data points) become spanned the less active growing zone emerge and thus sizes of compressed Physarum pattern decrease (Fig. 4f). Complexity of graph constructed increases almost linearly (Fig. 4f).

In this example with the model plasmodium, see Fig. 5, compressibility of the patterns (Fig. 5e) changes proportionally to (Fig. 5f) to edge-wise complexity 


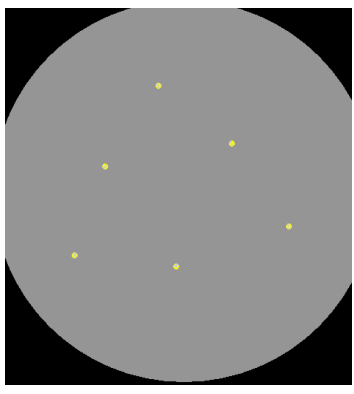

(a) $\mathrm{t}=10$

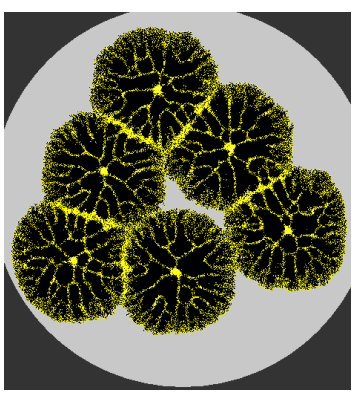

(d) $\mathrm{t}=150$

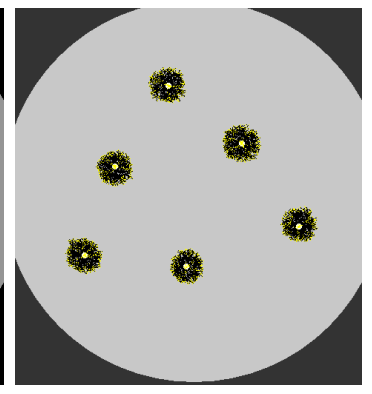

(b) $\mathrm{t}=60$

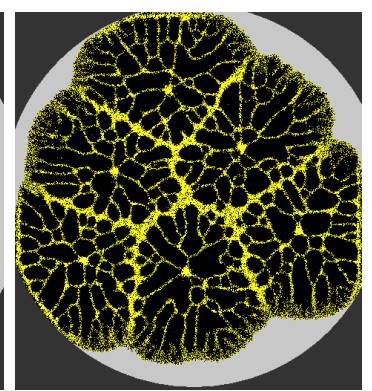

(e) $\mathrm{t}=210$

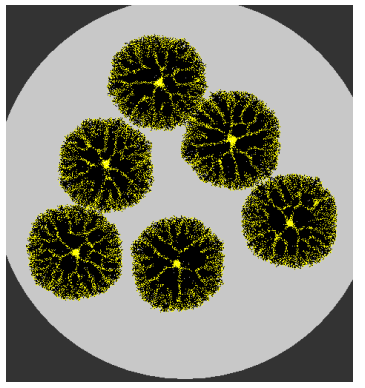

(c) $\mathrm{t}=120$

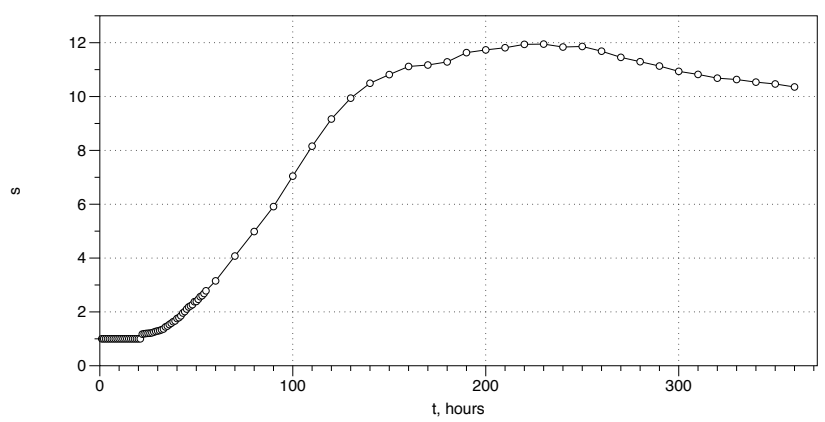

(g)

Figure 3: Approximation of Voronoi diagram by model plasmodium. (a) inoculation of seperate model plasmodia (yellow) on simulated oat flakes on nutrientrich background (grey), (b-c) Radial growth of model plasmodium, (d-e) Growth is temporarily inhibited at regions where other model plasmodia are occupied, These dense regions indicates bisectors of Voronoi diagram, (f) bisectors fade with continued adaptation, (g) dynamics of compressibility $s(t)$. 


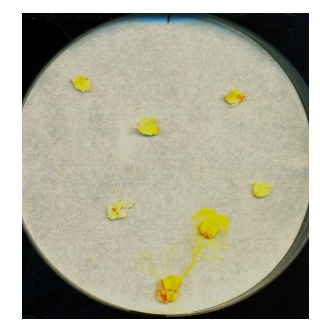

(a) $12 \mathrm{~h}$

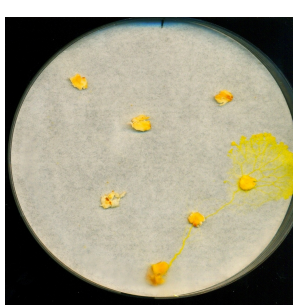

(b) $24 \mathrm{~h}$

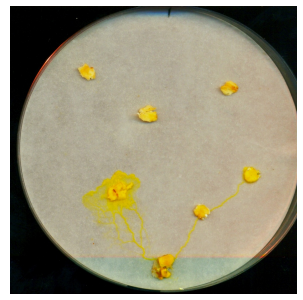

(c) $36 \mathrm{~h}$

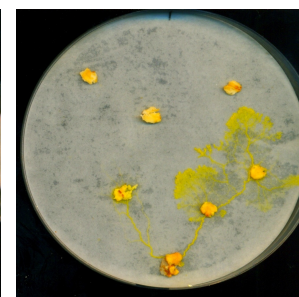

(d) $48 \mathrm{~h}$

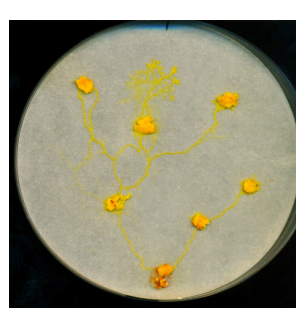

(e) $60 \mathrm{~h}$

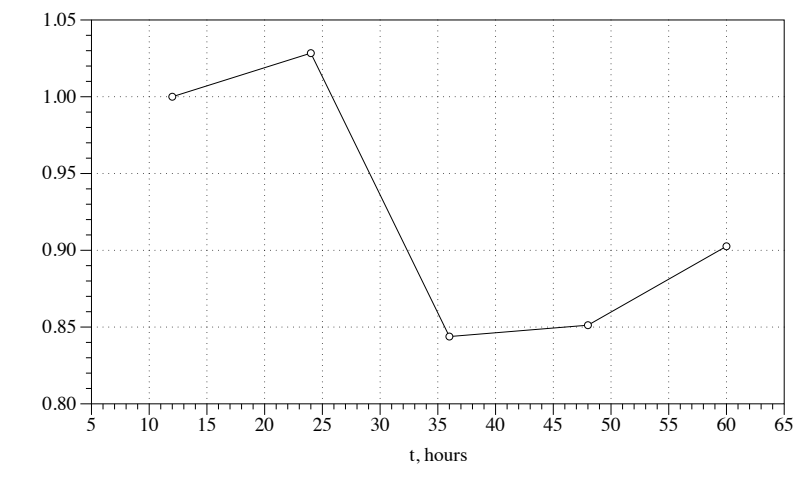

(f)

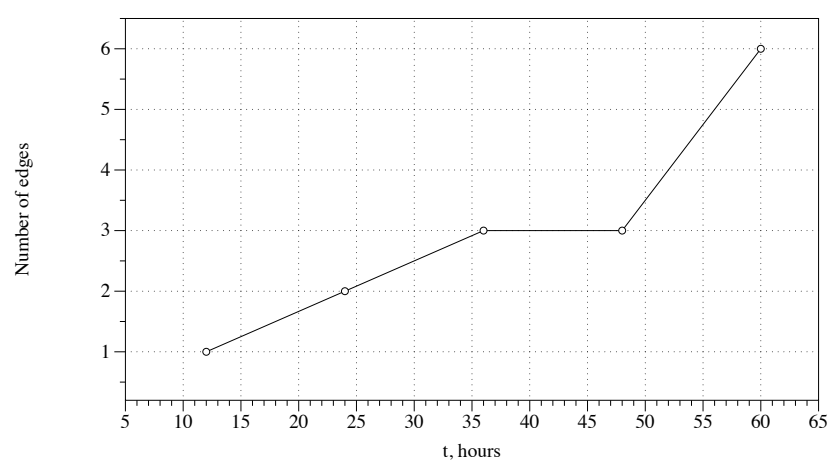

(g)

Figure 4: Physarum approximate spanning tree. (a-e) Photographs of the experimental setup taken in $12 \mathrm{~h}$ intervals. See details of setup in [2]. (f) Dynamic of compressibility $s(t)$. (g) Number of edges in the graph constructed. 


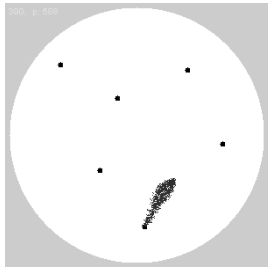

(a)

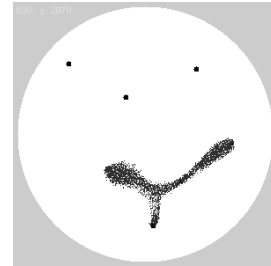

(b)

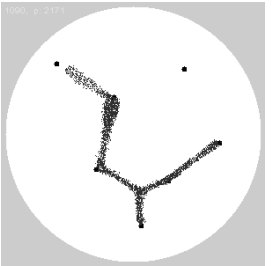

(c)

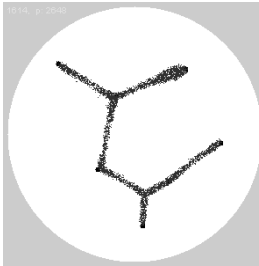

(d)

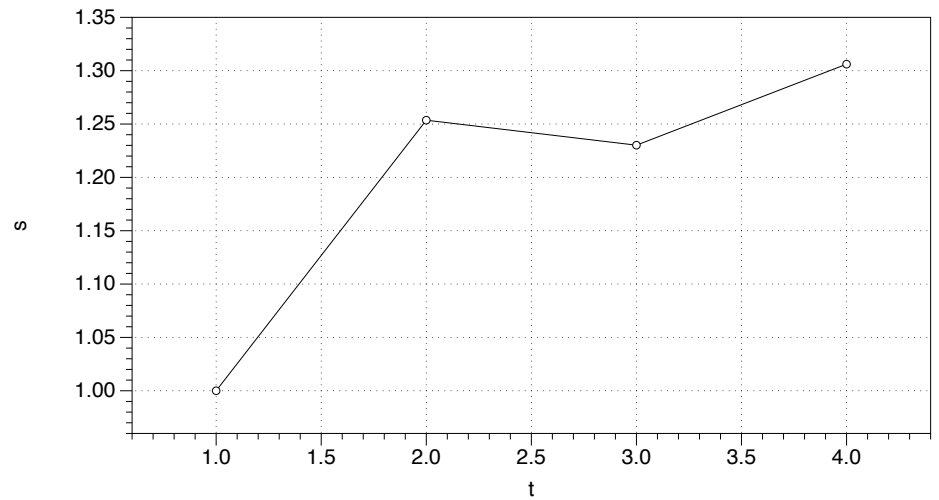

(e)

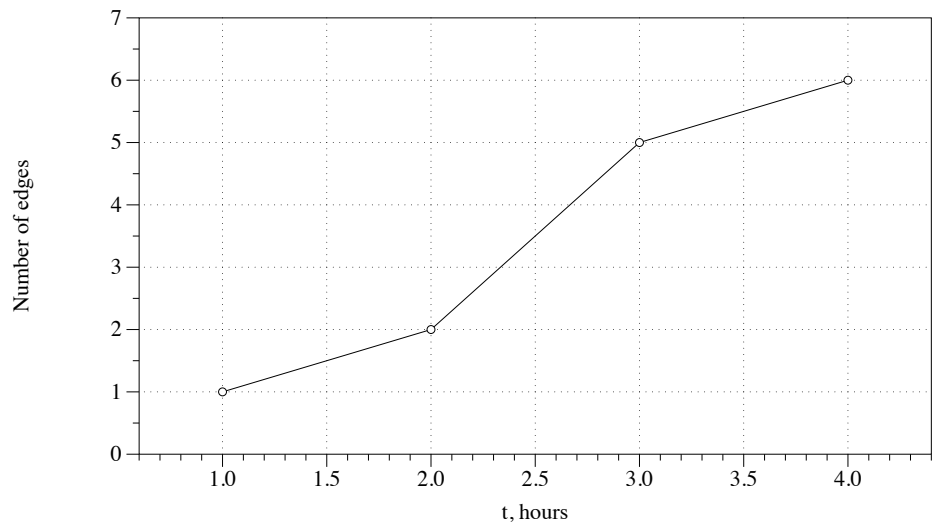

(f)

Figure 5: Modelling Physarum approximation of spanning tree. (a-e) Snapshots of the computer experiments. (f) Dynamic of compressibility $s(t)$. (g) Number of edges in the graph constructed. 
of the graph. We also investigated the behaviour of the model in response to more complex datasets. We randomly generated 20 points (corresponding to oat flakes) on a $300 \times 300$ lattice and inoculated the model plasmodium on a randomly chosen point (Fig. 6a, circled). We recorded images of the configuration of the model every ten steps and ran each experiment for 5,000 steps. A typical pattern of growth and adaptation is shown in Fig. 6a-f. From its inoculation site the model plasmodium extended pseudopodium-like processes to migrate towards and engulf each nutrient source. When all the nutrients were covered the resultant reduction in stimuli caused the shape of the population to adapt and minimise to conform to the shape of the points. We recorded the filesizes over ten experiments and the plot of dynamics of average $s(t)$ is shown in Fig. $6 \mathrm{~g}$ (thick line is aerage, standard deviation error bars indicated by shaded regions). The plot shows an increase in $s$ until all nodes are discovered (typically $\mathrm{t}=500-600$ steps). The adaptation phase is indicated by the downward slope (600-1000 steps) of $s$ and the stable configuration then continues and is represented by the relatively stable $s$ value.

To what extent does the increase in population size affect changing compressibility? It is possible to argue that the increase and decrease in file size (and subsequent decrease and increase in compressibility) is merely due to the increase and decrease in population and that the re-distribution of the plasmodium (real and virtual) does not affect compressibility. Using the model it is possible to have a 'fixed size' plasmodium which does not differ in the number of its components throughout an experimental run. Therefore any changes in compressibility must arise as a consequence of the self-organised network formation and adaptation. An example can be shown in Fig.7 in which we use a fixed population size of 4000 particles inoculated within a circular arena on a $200 \times 200$ lattice containing 50 nutrient sources. The population is initialised at random locations within the arena. Particle sensor parameters were SA 45 and RA 45 and SO was 7. Because growth and shrinkage of the population was disabled we reduced the concentration of the stimuli to 2.5 units per scheduler step and increased particle deposition to 5 units per step. This adjustment is necessary to prevent defects forming in the material from very high concentration stimuli.

Fig. 7 shows significant events in the evolution of the fixed sized model plasmodium. Initially there is no network (Fig. 7a) but a network composed of particles connecting the nutrient sources spontaneously forms (Fig. 7). This network undergoes minimisation over time, reducing the number of edges and cycles. The associated graph does not show the initial increase in $s(t)$ as the population size is fixed. Instead $s(t)$ decreases rapidly as the network forms and minimises. The evolution of $s(t)$ then stabilises over time, falling only at significant events in the network evolution (for example, when some remaining cycles are minimised, Fig. 7e,f,g and h). This result demonstrates that the changes in compressibility cannot be due only to changes in population size but also represent the inherent ordering of the networks over time.

\subsection{Maze}

A typical experiment is illustrated in Fig. 8, see details in [5]. We placed an oat flake in the central chamber and inoculated plasmodium of Physarum in a peripheral channels (Fig. 8a). The plasmodium started exploring its vicinity and at first generated two active zones propagating clock- and contra-clockwise 


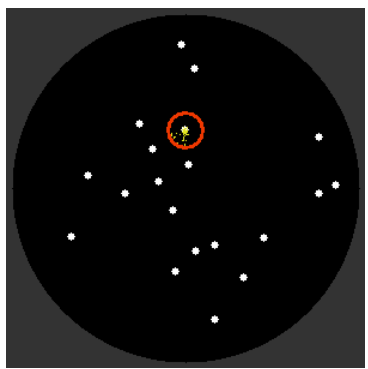

(a) $\mathrm{t}=50$

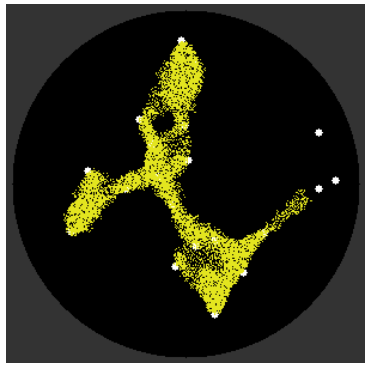

(d) $\mathrm{t}=370$

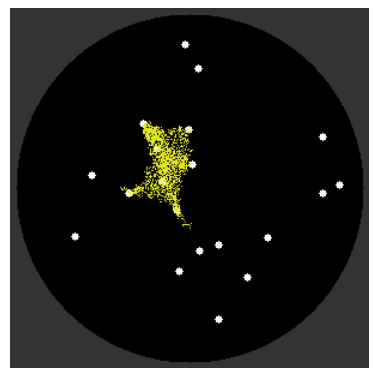

(b) $\mathrm{t}=130$

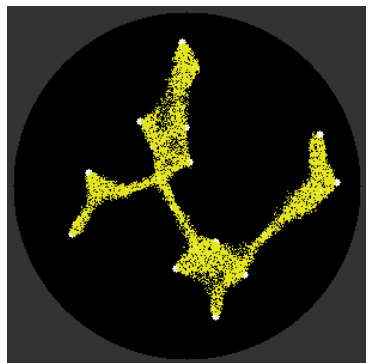

(e) $\mathrm{t}=560$

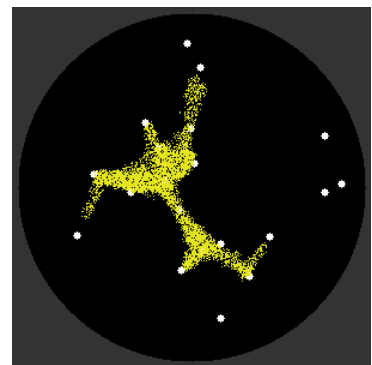

(c) $\mathrm{t}=240$

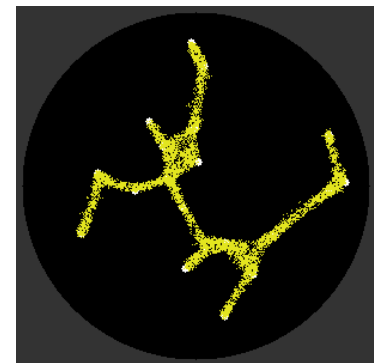

(f) $\mathrm{t}=5000$

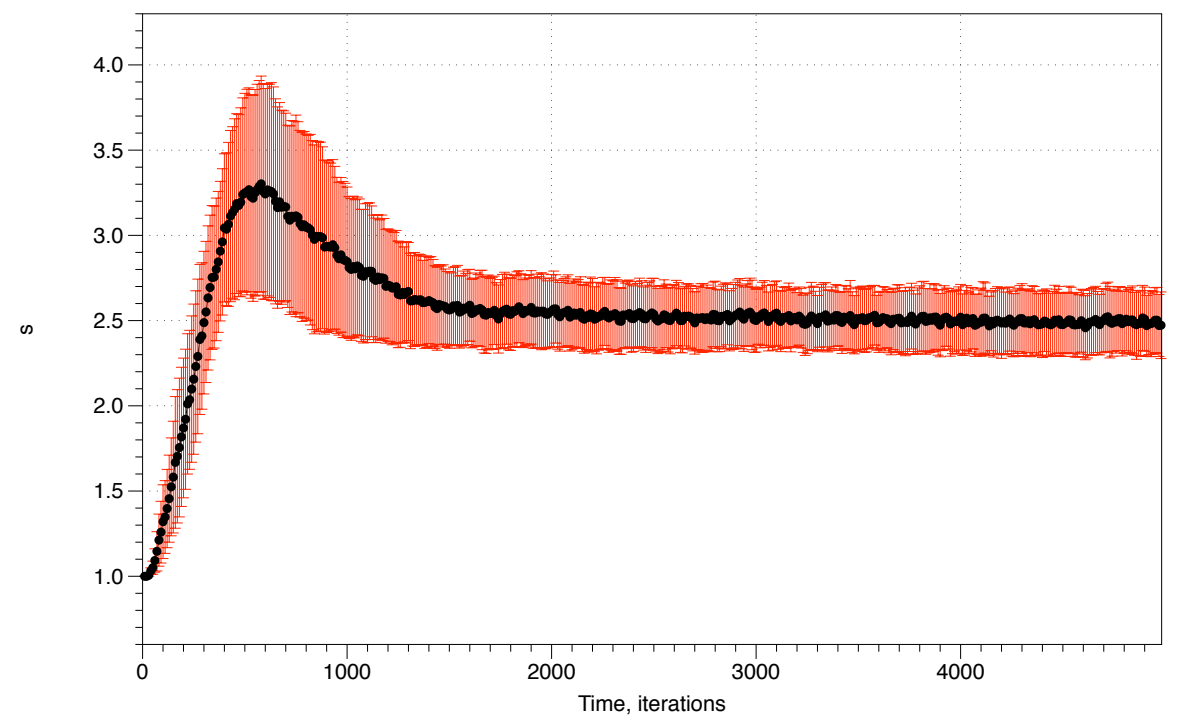

(g)

Figure 6: Dynamics of $s$ during construction and adaptation of spanning trees in the model plasmodium. (a) model plasmodium is inoculated on a randomly chosen site (circled) on a randomly generated $300 \times 300$ lattice of 20 data points, (b-f) Example evolution pattern of model plasmodium as it migrates towards sites, engulf sites and adapts to attractant profile, (g) plot of average dynamics of $s$ over 10 experiments (thick line) with standard deviation error bars indicated by shaded regions. 


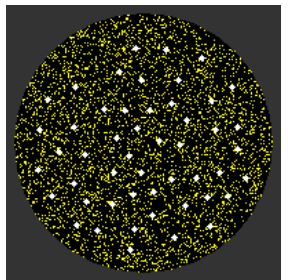

(a) $\mathrm{t}=1$

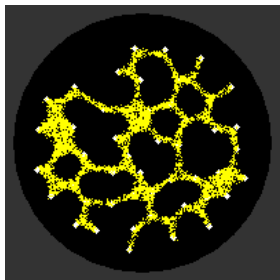

(e) $\mathrm{t}=5000$

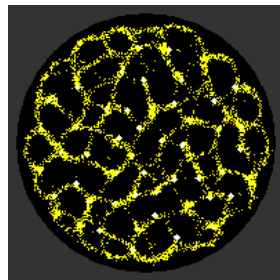

(b) $t=30$

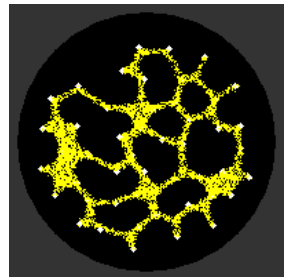

(f) $t=20000$

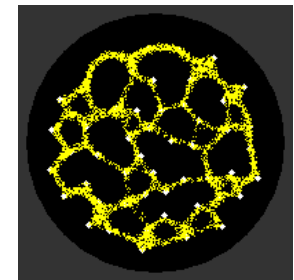

(c) $\mathrm{t}=400$

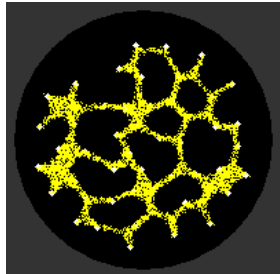

(g) $\mathrm{t}=27650$

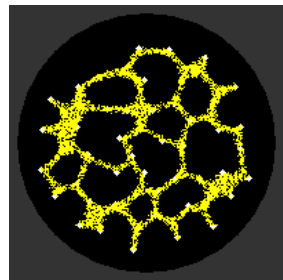

(d) $\mathrm{t}=1000$

Figure 7: Dynamics of $s$ during formation and adaptation of proximity graph in the model plasmodium. (a) model plasmodium of 4000 particles is inoculated at random sites within a $200 \times 200$ lattice of 50 data points, (b-h) Self-organised network formation and adaptation of model plasmodium as it adapts its shape to locations of attractants, (i) plot of dynamics of $s$ over experiment, initial rapid fall is followed by a long stable periods, punctuated only by removal of cycles within the network.

(Fig. 8bcd). By the time diffusing chemo-attractants reached distant channels one of the active zone already became dominant and suppressed another active zone (Fig. 8 efg). In example shown active zone travelling contra-clockwise dominated and 'extinguished' active zone propagating clockwise. The dominating active zone then followed gradient of chemo-attractants inside the maze, navigated along intersections of the maze's channels and solved the maze by entering its central chamber (Fig. 8hij). Spatio-temporal dynamic of space exploration by Physarum is well reflected in the dynamics of compressibility shown in Fig. 8k. Thus formation of branching active zones, or scouting branches at $10 \mathrm{~h}$ and $12 \mathrm{~h}$ of experiment, is reflected by peaks of compressibility $s$. When competition between active zones exploring various pathways ends in favour of one of the active growing zone, losers retract. These events lead to drops in compressibility, as e.g. in $12 \mathrm{~h}$ and $16 \mathrm{~h}$ of experimenting. When the Physarum finds the central chamber all scouting branches retract, or become abandoned, and the compressibility drops to its lowest level, indicating that the solution is found.

\subsection{Transport networks}

In experiments on analog modelling of the world colonisation discussed in [6] we represented major capitals of the world by virgin oat flakes and inoculated Physarum in Beijing (Fig. 9a). In first hours of the experiment Physarum propagates along South-East and South, colonising urban areas in India, Pakistan, Turkey (Fig. 9bcd). The function $s$ (Fig. 9l) exhibits peak at $6 \mathrm{~h}$. This corre- 


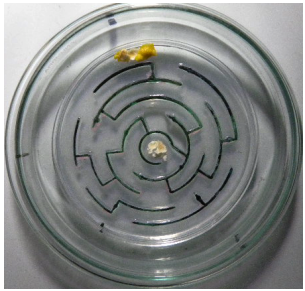

(a) $2 \mathrm{~h}$

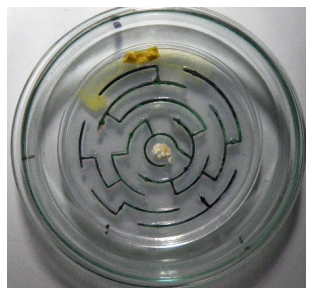

(d) $8 \mathrm{~h}$

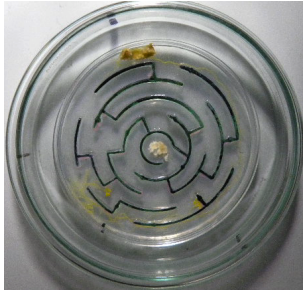

(g) $14 \mathrm{~h}$

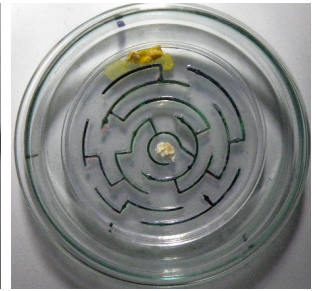

(b) $4 \mathrm{~h}$

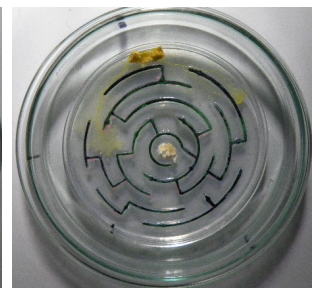

(e) $10 \mathrm{~h}$

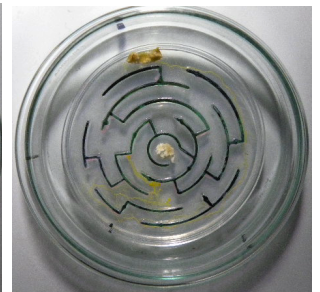

(h) $16 \mathrm{~h}$

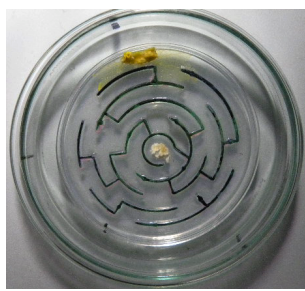

(c) $6 \mathrm{~h}$

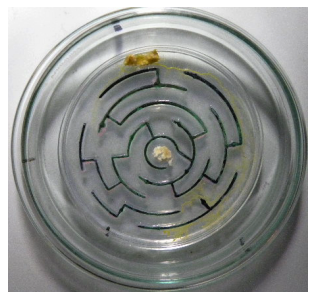

(f) $12 \mathrm{~h}$

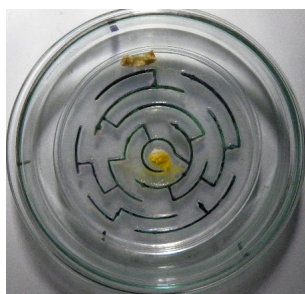

(i) $18 \mathrm{~h}$

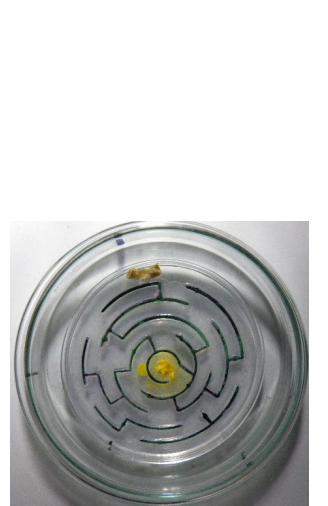

(j) $20 \mathrm{~h}$

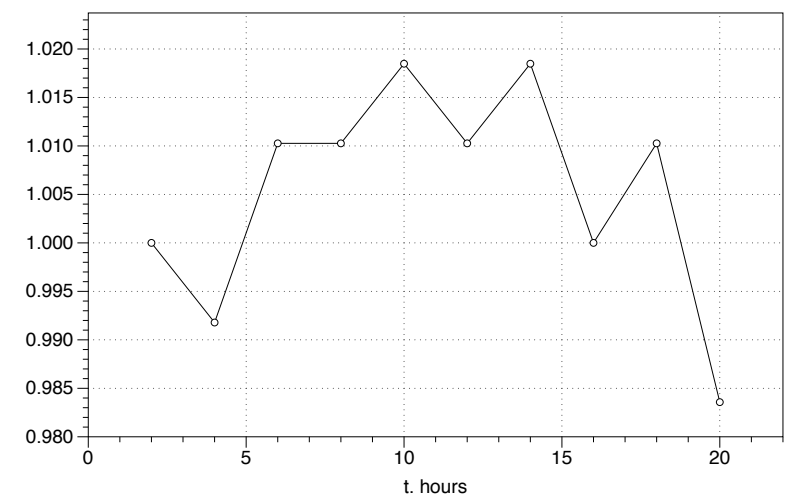

(k) $22 \mathrm{~h}$

Figure 8: Physarum solves maze problem (a-i) Photographs of the experimental setup taken in $2 \mathrm{~h}$ intervals. See details of setup in [5]. (k) Dynamic of compressibility $s(t)$. 


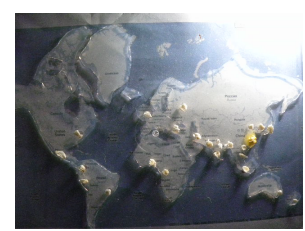

(a) $2 \mathrm{~h}$

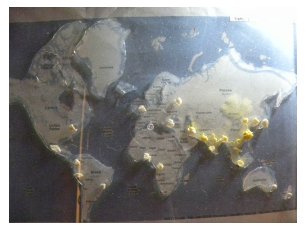

(e) $10 \mathrm{~h}$

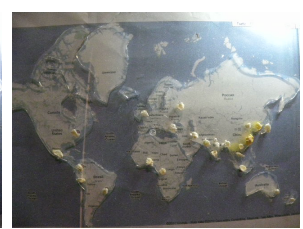

(b) $4 \mathrm{~h}$

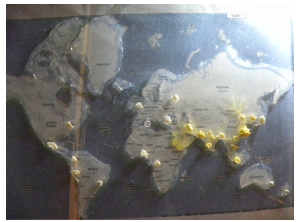

(f) $12 \mathrm{~h}$

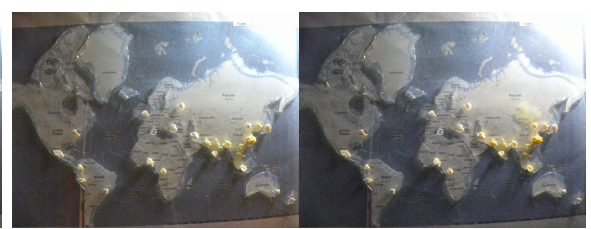

(c) $6 \mathrm{~h}$

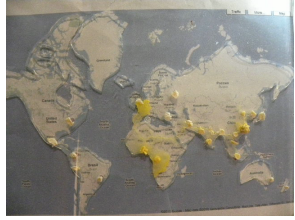

(g) $14 \mathrm{~h}$ (d) $8 \mathrm{~h}$

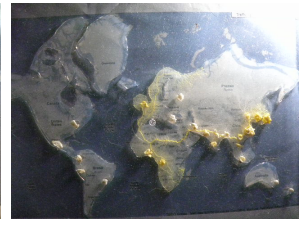

(h) $16 \mathrm{~h}$

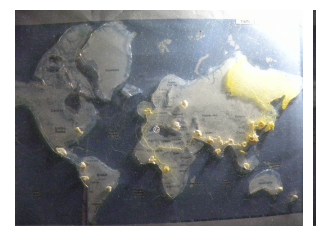

(i) $18 \mathrm{~h}$

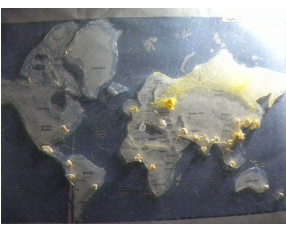

(j) $20 \mathrm{~h}$

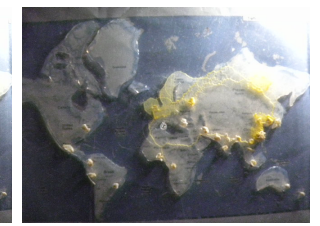

(k) $22 \mathrm{~h}$

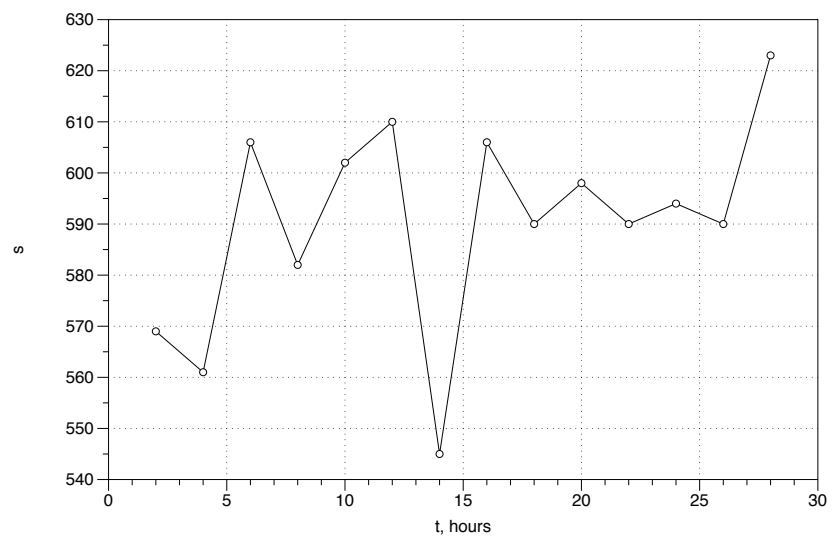

(1)

Figure 9: Physarum approximation the world transport networks. (a-k) Photographs of the experimental setup taken in $2 \mathrm{~h}$ intervals. See details of setup in [6]. (k) Dynamic of compressibility $s(t)$. 
sponds to increases activity of the slime mould: it propagates simultaneously from China to Japan and from India to Pakistan (Fig. 9c). At $10 \mathrm{~h}$ we can observe an scouting active zone sprouting through China to Russia (Fig. 9e); the active zone does not succeed and detracts back by $12 \mathrm{~h}$. Another peak of image size (decrease in compressibility) is observed at $12 \mathrm{~h}$ (Fig. 9l). This reflects developing of several pseudopodia spreading into Africa (Fig. 9f). The colonisation of Africa completes by $16 \mathrm{~h}$ (Fig. 9gh) which is manifested in substantial increase of compressibility, decrease of the image size (Fig. 9l). At $16 \mathrm{~h}$ also London is colonised and Physarum propagates into the North Europe (Fig. 9h) and we see corresponding peak in the image size: increased activity decreases compressibility. Another attempt, this time successful, to colonise Russian territory is made at $18 \mathrm{~h}$ (Fig. 9i). By $20 \mathrm{~h}$ the slime mould reaches Moscow (Fig. 9j) and recolonizes London again by $22 \mathrm{~h}$ (Fig. 9j). These activities do not change compressibility substantially because major part of Eurasia is already colonised by that time.

\section{Discussion}

Unconventional computers made of physical, chemical and living substrates rarely halt. Even when they find a solution to the given problem, they express their finding via morphological, chemical or electrical changes but then continue their 'daily business' as if nothing happened. Turing machine halts. Physarum machines never halt. We propose to use dynamics of compressibility of a system's spatial configurations to detect when the system completed computation. Compressibility of a system is inversely proportional to morphological diversity - a number of distinct local sub-configurations - of the system. When the system searchers for a solution of the problem it explores the space but also explores itself thus generating a vast number of different spatial sub-states. The patterns of the system in such states are difficult to compress. Compressibility of a system decreases when the system is looking for a solution. When the solution space exploration is abandoned the system returns to its state of minimal energy. Compressibility of such system increases. Thus by observing dynamics of the system's compressibility we can detect when the compressibility stabilised or increased to it maximum. We can conjecture that in that moment the system produces the solution to the problem.

Feasibility of our approach was tested on results of experimental laboratory and computer modelling studies of the slime mould computers. We found that when the slime mould $P$. polycephalum solves problems related to space filling (Voronoi diagram) or shortest path (maze solving) the maximum level compressibility indicates that solution is produced. This is somewhat analogous to behaviour of compressibility in other spatially extended systems performing computation:, e.g. cyclic tag systems emulated by cellular automata [24] or cellular automata solving a parity problem [22]. In scenarios when the slime mould must approximate a spanning tree or build transport networks stabilisation of compressibility dynamics is an indicator of completion of the computation. 


\section{References}

[1] Mateo Aboy, Roberto Hornero, Daniel Abásolo, and Daniel Álvarez. Interpretation of the Lempel-Ziv complexity measure in the context of biomedical signal analysis. Biomedical Engineering, IEEE Transactions on, 53(11):2282-2288, 2006.

[2] Andrew Adamatzky. Physarum machines: encapsulating reaction-diffusion to compute spanning tree. Naturwissenschaften, 94(12):975-980, 2007.

[3] Andrew Adamatzky. Physarum machines: computers from slime mould, volume 74. World Scientific, 2010.

[4] Andrew Adamatzky. On diversity of configurations generated by excitable cellular automata with dynamical excitation intervals. International Journal of Modern Physics C, 23(12), 2012.

[5] Andrew Adamatzky. Slime mold solves maze in one pass, assisted by gradient of chemo-attractants. NanoBioscience, IEEE Transactions on, 11(2):131-134, 2012.

[6] Andrew Adamatzky. The world's colonization and trade routes formation as imitated by slime mould. International Journal of Bifurcation and Chaos, $22(08), 2012$.

[7] Andrew Adamatzky and Leon O Chua. Phenomenology of retained refractoriness: On semi-memristive discrete media. International Journal of Bifurcation and Chaos, 22(11), 2012.

[8] Andrew Adamatzky and Genaro J Martinez. On generative morphological diversity of elementary cellular automata. Kybernetes, 39(1):72-82, 2010.

[9] Hussein Al-Bahadili and Ahmad Rababa'a. A bit-level text compression scheme based on the hcdc algorithm. International Journal of Computers 6 Applications, 32(3):355, 2010.

[10] José M Amigó, Janusz Szczepański, Elek Wajnryb, and Maria V SanchezVives. Estimating the entropy rate of spike trains via Lempel-Ziv complexity. Neural Computation, 16(4):717-736, 2004.

[11] Joydeep Bhattacharya et al. Complexity analysis of spontaneous EEG. Acta neurobiologiae experimentalis, 60(4):495-502, 2000.

[12] David P Feldman and Jim Crutchfield. A survey of complexity measures. Santa Fe Institute, USA, 11, 1998.

[13] P Preparata Franco, Michael Ian Shamos, and M Ian. Computational Geometry, an introduction. Springer-Verlag, New York, 1985.

[14] Jeff Jones. Characteristics of pattern formation and evolution in approximations of Physarum transport networks. Artificial Life, 16(2):127-153, 2010.

[15] Jeff Jones. The emergence and dynamical evolution of complex transport networks from simple low-level behaviours. Int. Journal of Unconventional Comput., 6:125-144, 2010. 
[16] Jeff Jones. From Pattern Formation to Material Computation: Multi-agent Modelling of Physarum polycephalum. Springer, in-press 2015.

[17] Jeff Jones and Andrew Adamatzky. Slime mould inspired generalised Voronoi diagrams with repulsive fields. Int. J. Bifurcation and Chaos, In-Press, 2013.

[18] Pavel G Khalatur, Viktor V Novikov, and Alexei R Khokhlov. Conformation-dependent evolution of copolymer sequences. Physical Review E, 67(5):051901, 2003.

[19] Toshiko Matsumoto, Kunihiko Sadakane, Hiroshi Imai, and Takumi Okazaki. Can general-purpose compression schemes really compress DNA sequences. Currents in Computational Molecular Biology, pages 76-77, 2000 .

[20] Toshiyuki Nakagaki. Smart behavior of true slime mold in a labyrinth. Research in Microbiology, 152(9):767-770, 2001.

[21] Jaroslav Nešetřil, Eva Milková, and Helena Nešetřilová. Otakar Boruvka on minimum spanning tree problem translation of both the 1926 papers, comments, history. Discrete Mathematics, 233(1):3-36, 2001.

[22] Shigeru Ninagawa. Solving the parity problem with Rule 60 in array size of the power of two. arXiv preprint arXiv:1307.3888, 2013.

[23] Shigeru Ninagawa and Andrew Adamatzky. Classifying elementary cellular automata using compressibility, diversity and sensitivity measures. International Journal of Modern Physics C, 25(03), 2014.

[24] Shingeru Ninagawa and Genaro J Martinez. Compression-based analysis of cyclic tag system emulated by Rule 110. J. Cellular Automata, 9(1):23-35, 2014.

[25] Yuri L. Orlov and Vladimir N Potapov. Complexity: an internet resource for analysis of DNA sequence complexity. Nucleic acids research, 32(suppl 2):W628-W633, 2004.

[26] Markus Redeker, Andrew Adamatzky, and Genaro J Martínez. Expressiveness of elementary cellular automata. International Journal of Modern Physics C, 24(03), 2013.

[27] Steven L Stephenson, Henry Stempen, and Ian Hall. Myxomycetes: a handbook of slime molds. Timber Press Portland, Oregon, 1994.

[28] Jacob Ziv and Abraham Lempel. Compression of individual sequences via variable-rate coding. Information Theory, IEEE Transactions on, 24(5):530-536, 1978. 